\title{
Guarcs in the Inside Hadronic Four-Dimensional Euclidean Space with Real Time
}

\section{Eugene Kreymer*}

Institute for Physics and Engineering, Donetsk, 83114, Ukraine

\begin{abstract}
The paper represents the results of the study of the four-dimensional Euclidean space with real time (E-space), where $0 \leq\|V E\| \leq \infty$, in sub-hadronic physics. This closed space has a metric that distinguished from the Minkowski space and the results obtained in the model are different from physical law in the Minkowski space. As it follows from the model of Lagrangian Mechanics, quarks in the central-symmetric attractive potential, kinetic energy of quark diminishes while the speed grows as the quarks exchange their energy-mass with gluons possessing a zero rest mass, so that to ensure the permanent proton mass. This dependence describes the dynamical relation of constituent and current quarks masses.

In the quantified motion model it has been stated, that the oscillations of the particles are cyclic, including alternating localization and translation phases, the action per cycle for a free particle equals $\overline{\mathrm{h}}$. The calculation of charge distribution density in proton, carried out on the basis of this model, conforms to the results of the experimental research. All relations between physical values in the E-space, mapped in the Minkowski space, correspond to the principles of SR and are Lorentz-covariant and the infinite velocity is equal to the velocity of light in the Minkowski space. These models have a transparent physical sense.
\end{abstract}

Keywords: Dynamics of quarks in the proton; Euclidean invariants; Motion of quarks and gluons; Quantum cyclic motion; Charge distribution in the proton

\section{Introduction}

Non-perturbative effects are of great importance for the theory of space inside hadron. Supposing a sequence of QCD problems are concentrated in the branch of occurrences that can be described through the transition from the Minkowski space $\mathrm{M}\left(x_{M 0}, x_{M 1}, x_{M 2}, x_{M 3}\right)$ (M-space) into the Euclidean space inside hadron via the analytical extension of the time axis onto the lower semi plane $x_{E i 0}=i x_{\mathrm{M} 0}$. In this case we get the Euclidean space with the imaginary time $\mathbf{E}_{i m}\left(x_{E i 0}, x_{E 1} x_{E 2}, x_{E 3}\right)\left(\mathbf{E}_{i m}\right.$ is space), and $\mathbf{X}_{E i=} \mathbf{X}_{M}$ is automatically $\mathbf{V}_{E i}=i \mathbf{V}_{M}$ and $\boldsymbol{0} \leq\left\|\boldsymbol{V}_{E i}\right\| \leq \mathbf{1}$. The use of such a space has brought to great results: the QCD valuum models, lattice calculations, string theory and so on. However, e.g. QCD in lattice can now be used only for the description of a limited class of hadronic elements of the matrix. There is no common and self-congruent description of the QCD vacuum heretofore, as well as confinement occurrence and a spontaneous disturbance of the chiral invariance. In the common case the rotation group of the Euclidean space in the plane $\left(x_{E 0}, \mathbf{X}_{E}\right)$ presupposes that $\mathbf{0} \leq\left\|V_{E}\right\| \leq \infty$, while $\mathbf{E}_{i m} \mathbf{0} \leq\left\|V_{E i}\right\| \leq \mathbf{1}$. At the same time $\mathbf{E}_{i m}$ is not even a subspace of the Euclidean space, because it is not closed in respect of the operation of composition of vectors. Thus, an infinite velocity causing non-local (instantaneous) interactions and contained in some NQCD models lies outside the frames of $\mathbf{E}_{i m}$ - space. Non-local quark non-perturbative vacuum condensate plays a crucial role while creating realistic hadrons models [1]. At the same time the space correlation functions look like the curve of decreasing exponent [2] whose negative parameters include the distance of $z=x-y$ while $x_{E i 0=}$ const.

In correspondence with $[3,4]$ physics of non-locality starts to be seen at the distance of $\lambda \approx 0,2 \mathrm{fm}$. The correlation length $\lambda$ determines the spatial declining of bound gauge-invariant bilocal correlator of field gradient.

In other studies, a minimal Gauss model, offered in [5], is used for condensates in a non-perturbative vacuum. The parameter of non- locality $\lambda$ characterizes an average square of quarks' impulse in the $\mathrm{QCD}$ vacuum. Its estimations by means of $\mathrm{QCD}$ in lattice have shown the following range of probable values: $\lambda_{q}^{2}=0.45 \pm 0.1 \Gamma \ni \mathrm{B}^{2}[6,7] . \mathbf{E}_{i m}$ - is homomorphic in respect of the $\mathrm{M}$-space and non-local, in other words, the instantaneous interactions even at some low $\lambda$ value contradict with $\mathrm{S}$ principles.

It gives a reason to consider that the use of merely a part of fourdimensional Euclidean space volume in the models with $\mathbf{E}_{\text {im }}$ does not allow using its potential to the full extent. The article expounds the first steps in the research of the inside hadronic four-dimensional Euclidean space with real time model $\mathrm{E}\left(x_{E 0,}, x_{E 1}, x_{E 2}, x_{E 3}\right)$ (E-space), where $\boldsymbol{0} \leq$ $\left\|V_{E}\right\| \leq \infty$, and its aim is to show the expedience of the studies in the E-space as s probable prospective direction of sub-hadronic physics development. The article contains researches of the E-space properties in protons and it is presupposed that the obtained correlations have a common nature and can cover all the hadrons. Moreover it has been considered been considered that the models in the $\mathbf{E}$-space will not be an alternative for the theoretical developments in $\mathbf{E}_{i m}$, but will extend their possibilities. The following requirement is the basic condition enabling this model to exist:

Requirement 1: Space-time relations and regularities in the E-space model mapped into the $\mathbf{M}$-space must correspond to the principles of SR and be Lorentz-covariant.

*Corresponding author: Eugene Kreymer, Institute for Physics and Engineering,
Donetsk, 83114, Ukraine, Tel: (062) 311-52-27; E-mail: elkreymer@gmail.com

Received February 28, 2015; Accepted June 09, 2015; Published June 16, 2015

Citation: Kreymer E (2015) Guarcs in the Inside Hadronic Four-Dimensional Euclidean Space with Real Time. J Phys Math 6: 140. doi:10.4172/20900902.1000140

Copyright: (c) 2015 Kreymer E. This is an open-access article distributed under the terms of the Creative Commons Attribution License, which permits unrestricted use, distribution, and reproduction in any medium, provided the original author and source are credited. 


\section{Inside Hadronic Euclidean Frames of Reference}

In the E-space no frames of reference, which are microscopic in reality, can be physically implemented. To determine the spatial coordinates the laboratory frame of reference LFR with the coordinates $\left(x_{\mathrm{M} 0}, x_{\mathrm{M} 1}, x_{\mathrm{M} 2}, x_{\mathrm{M} 3}\right)$ has been used, where hadron rests, and $d \mathbf{x}_{\mathrm{E}=} d \mathbf{x}_{\mathrm{M}}$. The own time of particles in the LFR is admitted to be the temporal coordinate $x_{E O,}$ Thus the $\mathbf{E}$-space is "subsidiary" towards the $\mathbf{M}$-space.

Definition 1: Inner hadronic four-dimensional Euclidean Frame of Reference $\left(x_{E 0,} x_{E 1}, x_{E 2}, x_{E 3}\right) \mathrm{EFR}$, is a system, where the space coordinates are indexed by the coordinates of $\left(\mathrm{x}_{\mathrm{M} 1}, \mathrm{x}_{\mathrm{M} 2}, \mathrm{x}_{\mathrm{M} 3}\right)$ LFR and the own time of the particles is equal to the own time of the particles in the LFR

$$
d x_{E 0 i}=d s_{M i}=d x_{M 0 i} \sqrt{1-\mathbf{v}_{M i}^{2}},
$$

Where $\mathrm{V}_{\mathrm{Mi}}$ is the velocity of the i-number particle in the LFR. The transition to the other IFR is carried out by means of Lorenz transformation. The $\mathbf{E}$-space of the real particles corresponds to the $\mathbf{M}$-space upper closed cone $\overline{\mathrm{V}}_{+}:=\left\{x_{M} \in \mathrm{M} / x_{M}^{2} \geq 0,+x_{M 0} \geq 0\right\}$ and $\mathrm{X}_{\mathrm{E} 0}=[0 ; \infty)$, that ensures the execution of the causality principle. The EFR has an invariant which taking into consideration the Definition 1 is equal to

$$
d x_{E 0}^{2}+d \mathbf{x}_{E}^{2}=d x_{M 0}^{2}
$$

Then there is symmetry between EFR and LFR: the time of one space is the invariant of the other.

From (2.1) and (2.2) it follows that

$$
\mathbf{v}_{M}=\frac{\mathbf{v}_{E}}{\sqrt{1+v_{E}^{2}}},
$$

Where $\mathbf{v}_{\mathrm{E}}$ - is the velocity of the particle in the EFR. And, correspondingly

$$
\mathbf{v}_{E}=\frac{\mathbf{v}_{M}}{\sqrt{1-v_{M}^{2}}} .
$$

If $\mathbf{v}_{\mathrm{E}} \rightarrow \infty$, then $\mathbf{v}_{\mathrm{M}} \rightarrow 1$. There is also 4 -vector of velocity in the EFR

$$
u_{E}=\left(\frac{1}{\sqrt{1+v_{E}^{2}}} ; \frac{\mathbf{v}_{E}}{\sqrt{1+v_{E}^{2}}}\right)
$$

And its invariant is equal to the invariant of the corresponding relativistic 4-vector.

$S O(2)$ Group of the rotation of plane $\left(x_{E 0,} \mathbf{x}_{\mathrm{E}}\right)$, cannot be applied in the EFR, because the existence of the infinite velocity makes the time absolute, and $x_{E 0}$ can take no negative values. In accordance with (2.2), E-group of position-vector rotations $\mathrm{E}$ which describes the particles moving with different velocity is valid in the EFR. This group does not mix the temporal $x_{E 0}$ and the spatial coordinates $\mathrm{R}_{E(}\left(x_{E 1}, x_{E 2}, x_{E 3}\right)$. Mapping kinetic parameters of the particle in ERF observed in into LRF putting the fundamental quadratic forms

$$
\begin{aligned}
& {[\mathrm{E}]=\left(d x_{E \mu}, g_{E \mu \nu} d x_{E v}\right)=d s_{E}^{2}, \text { where } g_{E \mu \nu}-\text { Kronecker symbol, } \mu, v=0,1,2,3 \text { and }} \\
& {[M]=\left(d x_{M \mu}, g_{M \mu v} d x_{M v}\right)=d s_{M}^{2}, \text { where } g_{M \mu v} \text {-metric tensor. The translation }}
\end{aligned}
$$
matrix

$$
\varphi_{E M}:[E] \mapsto[M]=\left(d x_{E}^{o}, g_{E} d x_{E}\right) \mapsto\left(\mathrm{K}_{\mathrm{EM}} \mathrm{x}_{\mathrm{E}}^{\dot{o}}, \mathrm{G}_{\mathrm{EM}} \mathrm{g}_{\mathrm{E}} \mathrm{K}_{\mathrm{EM}} \mathrm{x}_{\mathrm{E}}\right)=\left(d x_{M}^{\dot{o}}, g_{M} d x_{M}\right)
$$

must involve kinematic $K_{E M}$ and metric $G_{E M}$ transformations. With (2.2) we obtain the kinematic transformation matrix $\left\|K_{\dot{E}}\right\|=\operatorname{diag}\left(\sqrt{1+\mathbf{v}_{E}^{2}}, 1,1,1\right)$. the metric transformation matrix $\left\|\mathrm{G}_{\mathrm{EI}}\right\|=$ diag $(1,-1,-1,-1)$. There is a distinction of properties of the studied space from the Minkowski space that emerges because of different metric: E-group of radius-vector rotations $x_{M 0}$ does not mix the temporal $x_{E 0}$ and spatial coordinates $\mathrm{R}_{\mathrm{E}}\left(x_{E 1}, x_{E 2}, x_{E 3}\right)$.

\section{The Model of E-invariant Lagrange Mechanics Particle}

\section{4-vector energy- momentum}

Lagrange function of the free particle

$$
L_{E}=m \sqrt{1+\left(\mathbf{v}_{E}\right)^{2}} \text {. }
$$

The momentum of the particle

$$
\mathbf{p}_{E}=\frac{m \mathbf{v}_{E}}{\sqrt{1+\mathbf{v}_{E}^{2}}},
$$

And the kinetic energy

$$
E_{E}=L_{E}-\mathbf{v}_{E} \frac{\partial L_{E}}{\partial \mathbf{v}_{E}}=\frac{m}{\sqrt{1+\mathbf{v}_{E}^{2}}} .
$$

This equation is valid under condition that $E_{E} \geq 0$. . At the same time

$$
\mathrm{E}_{\mathrm{E}+}^{2} \mathrm{P}_{\mathrm{E}}^{2}=\mathrm{m}^{2}
$$

From (3.4) we can make a conclusion that there is a 4 -vector of energy-momentum in the EFR, and its invariant is equal to the invariant of the corresponding relativistic 4 -vector and it is one more symmetry between the LFR and EFR. Translating the 4-vector of the particle in LFR through (2.3), we obtain $E_{E M}=m \sqrt{1-\mathbf{v}_{M}^{2}}$ и $\mathbf{p}_{E M}=m \mathbf{v}_{M}$. These values stay $\mathbb{E}$-invariant.

Formula (3.3) testifies to an unusual behavior in the $\mathbf{E}$-space of the kinetic energy: it diminishes when the speed grows. The next unit will demonstrate that it is so because of the energy-mass exchange between quarks and gluons.

\section{Mechanics of quark in the proton}

Here we use the model where quarks are considered electrically neutral particles, and we admit that in the center of a proton there is a hypothetical source creating central-symmetrical attractive potential $\mathrm{V}(r)$ of strong interactions. It is considered that this simplified model will provide the possibility to determine some peculiarities of quarks motion in the proton. $\mathrm{V}(r)$

The E-invariant Lagrange function of the quark in the potential

$$
L_{E V q}=m_{q} \sqrt{1+\mathbf{v}_{E q}^{2}}-\mathrm{V}(r),
$$

where $m_{q}$ - constituent mass of the quark. E-invariance of this function is ensured by $d \mathbf{x}_{E}=d \mathbf{x}_{M}$ potential $\mathrm{V}(r)$ will be identical for each proton in LFR.

On the analogy with (3.3) the energy of the system "quark potential $\mathrm{V}(r)$ »

$$
\mathrm{E}_{\mathrm{V}}=\mathrm{E}_{\mathrm{Eq}}-\mathrm{V}(r)=\text { const. }
$$

If a particle is under the influence of power $\mathbf{F}=-\nabla \mathrm{V}(r)$ parallel to the velocity, that it will change the momentum as follows:

$$
\frac{d \mathbf{p}_{E q}}{d x_{E 0}}=\frac{m_{q}}{\left(1+\mathbf{v}_{E}^{2}\right)^{\frac{3}{2}}} \frac{d \mathbf{v}_{E q}}{d x_{E 0}}=\mathbf{F} .
$$

The alteration of the energy

$$
\frac{d E_{E q}}{d x_{E 0}}=-\frac{\mathbf{v}_{E q} m_{q}}{\left(1+\mathbf{v}_{E q}^{2}\right)^{\frac{3}{2}}} \frac{d \mathbf{v}_{E q}}{d x_{E 0}}=-\mathbf{F} \mathbf{v}_{E q} .
$$


From which

$$
d \mathbf{p}_{E q}=\mathbf{F} d x_{E 0}(a) ; \quad d E_{E q}=-\mathbf{F} \mathbf{v}_{E q} d x_{E 0}=\nabla \mathrm{V}(\mathbf{x})(b) .
$$

From the (3.9b) and (3.6) it follows that $E_{\mathrm{V}}=0$. The zero-value of $E_{\mathrm{V}}$ is a result of the fact that gluons have not been taken into account. To ensure the constant proton mass, the alteration of the quark kinetic motion must be compensated by the relevant alteration of gluons energy - mass. Taking gluons into account.

$\mathrm{L}_{\mathrm{EV}}=\mathrm{L}_{\mathrm{Eq}}\left(\mathbf{v}_{\mathrm{q}}\right)+\mathrm{L}_{\mathrm{EG}}\left(\mathbf{v}_{\mathrm{G}}\right)-\mathrm{V}(r)$, where $\mathrm{L}_{\mathrm{EG}}\left(\mathbf{v}_{\mathrm{G}}\right)$ is the Lagrange function for gluons. makes

The preserved energy of "quark - gluon - potential V(r) system

$$
E_{V q G}=L_{E q}\left(\mathbf{v}_{q}\right)-\mathbf{v}_{q} \frac{\partial L_{E q}\left(\mathbf{v}_{q}\right)}{\partial\left(\mathbf{v}_{q}\right)}+\mathbf{v}_{G} \frac{\partial L_{E G}\left(\mathbf{v}_{G}\right)}{\partial\left(\mathbf{v}_{G}\right)}-L_{E G}\left(\mathbf{v}_{G}\right)-\mathrm{V}(r)=m_{q} .
$$

This equation has a solution, if $\mathbf{v}_{\mathrm{G}}=\mathbf{v}_{q}$. Then in the potential $\mathrm{V}(r)$

$$
L_{E G V}=m_{q} \sqrt{1+\left(\mathbf{v}_{q}\right)^{2}}-\mathrm{V}(r)-m_{q}
$$

Gluon momentum is

$$
\mathbf{p}_{E G}=\frac{m_{q} \mathbf{v}_{q}}{\sqrt{1+\mathbf{v}_{q}^{2}}},
$$

And the energy

$$
E_{E G}=m_{q}-\frac{m_{q}}{\sqrt{1+\mathbf{v}_{q}^{2}}} .
$$

From Esq. (3.3) and (3.13) we can draw a conclusion, that the energy - mass of the quark translates into the energy - mass of the gluon, and their sum makes equals $m_{\mathrm{q}}$. At the same time $\mathbf{P}_{\mathrm{EG}}=\mathbf{P}_{\mathrm{Eq}}$ and gluons are moving along with quarks creating valon. As a result, the constituent mass of quarks includes zero rest mass. This determines the dynamical relation of constituent and current quarks' masses. The quark mass diminishes as it approaches to the centre of a proton. This corresponds to the existing idea that quark has a minimum mass under a big transferred to it $q^{2}$ momentum. There are some scientific studies devoted to the NQCD, in which gluons are described as possessors of dynamical energy - mass [8]. Contains an approximate solution of Dyson-Schwinger equation, where a propagator of non-perturbative gluon is regulated by the dynamical generated mass of a gluon. The usage of this propagator gives an opportunity to calculate sections of pp- scattering and achieve a good concord of calculations with experimental data for an effective gluon mass of $370 \mathrm{MeV}$ [9], this value corresponds to $m_{\mathrm{q}}$ in the nucleon. The fact that gluon has peculiarities of a massive particle is confirmed by calculations in lattice $[10,11]$. In the papers $[12,13]$ different non-zero masses of gluons have also been studied. Let us examine the quark motion in the linearly increasing potential $\mathrm{V}(r)=c r$. The zero orbital moment of a proton along with experimental studies of the charge distribution in proton means that the quark is vibrating along the diameter towards the center of a proton. Let us presuppose that the quark vibrates under the power of $\left|\mathrm{F}_{\mathrm{z}}\right|=$ constant along the $z$ axis which has a null in of the center a proton. Basing $n$ the eq. (3.2), (3.3) and (3.9a) we obtain

$$
\frac{d z}{d x_{E 0}}=\frac{p_{E z q}}{E_{E q}}=\frac{F_{z} x_{E 0}}{\sqrt{m_{q}^{2}-F_{z}^{2} x_{E 0}^{2}}}=\frac{a_{\mathrm{clz}} x_{E 0}}{\sqrt{1-\left(a_{\mathrm{cl} 2} x_{E 0}\right)^{2}}},
$$

Where $a_{\mathrm{clz}}=F_{z} / m_{q}$ is a "classic" acceleration? Then

$$
z=-\sqrt{a_{\mathrm{clz}}^{-2}-x_{E 0}^{2}}
$$

and

$$
v_{E z q}=\frac{\sqrt{a_{\mathrm{clz}}^{-2}-z^{2}}}{z} \text {. }
$$

The dependence of the quark energy on the radius is $E_{E q}=m z a_{\mathrm{clz}}^{-1}$. From the (3.6) we can draw a conclusion that $\mathrm{V}\left(r_{\max }=r_{p}\right)=m_{q}$, where $r_{p}$ is the radius of a proton and $a_{\mathrm{clz}}^{-1}=r_{p}$. Under the condition that $-a_{c l z}^{-1} \leq x_{E 0} \leq a_{c l z}^{-1}$ in the coordinates $\left(x_{E 0}, z\right)$ quark makes a circumference with a radius $a_{\mathrm{clz}}^{-1}$. But the allowable values are $x_{E 0}=[0 ; \infty)$ and this formula must be specified. The half period of quark vibration is $\theta=2 a_{c l z}^{-1}$ and to preserve $x_{E O}$ in the given range of values we need to put (3.15) it in the following way:

$$
z= \pm \sqrt{a_{\mathrm{clz}}^{-2}-\left(t_{E 0}-a_{\mathrm{clz}}^{-1}\right)^{2}},
$$

Where $t_{E 0}=x_{E 0}-[n] 2 a_{\mathrm{clz}}^{-1}, \quad[n]$ is the biggest whole number in $x_{E 0} / 2 a_{c l z}^{-1}$. The digits before the root take turns depending on the alteration of $[n]$.

Thus, a vibrating quark makes two half circumferences with $z>0$ and $z<0$, moved at $2 a_{\mathrm{clz}}^{-1}$. Figure 1 shows the graph of the quark oscillations. The calculation involves the rms radius of the proton $r_{p}=0.84 \mathrm{fm}$.

Here we can show how the formula (3.6) is functioning. Under $z=0$ and $\mathrm{V}=0$ the speed makes $v_{E z q}=\infty$ and $E_{E q}=0$ (points A, C, E). Under $z= \pm a_{\mathrm{clz}}^{-1}$ and $v_{E z q}=0$ as well as $E_{E q}=m_{\mathrm{q}}$, as well as $\mathrm{V}=m_{q}$ (points B, D). And therefore $E_{E q}-\mathrm{V}=0$.

This brings up a question: how do the oscillations of quarks provide total zero momentum in the motionless proton while they are oscillations? Under multi-particle interactions, a symmetric disposition of particles corresponds to the minimum of energy and therefore a proton possesses a spherical symmetry and that means that 3 quarks make diametric oscillations creating a space angle $\pi$ and their impulses are getting balanced. This supposition correlates with analytical studies described in [14]; according to them effective fields in baryons has a Y-shaped configuration of quarks' plane making an equilateral triangle. This conclusion has also been confirmed by calculations in lattice [15].

\section{Models of E-Invariant Quantized Motion of Massive Particles}

A peculiarity of inside hadronic E-space is that its size in the three-

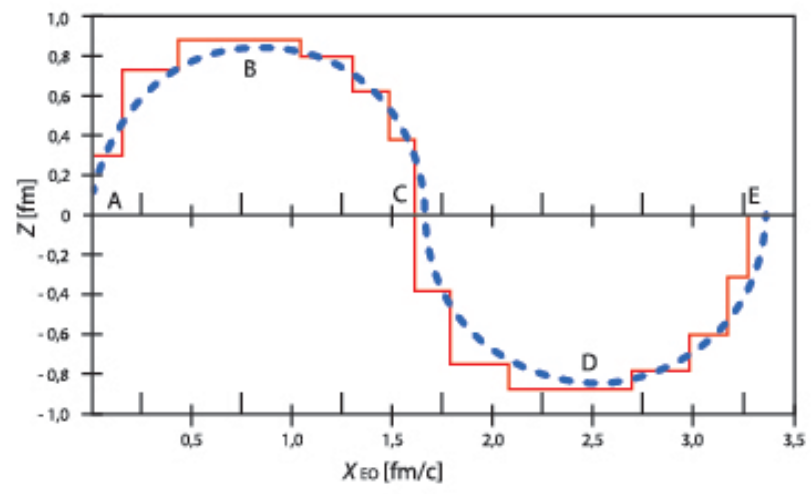

Figure 1: Diametrical quark oscillations in the proton: dash line is for the model of Lagrangian Mechanics, continuous line is for the model of quantified motion: points $A, C, E-E z q v=\infty, 0$; points $B, D-E E q=0$ Ezq $v=\infty, E m$ Eq q $c=1$. 
dimensional space is comparable to the Compton quark wave length and the maximum value of quark kinetic energy makes $m$. According to the quantum mechanics the minimum quark energy in the limited space must excess its mass. This is also applicable for oscillators' energy in the quantum field theory. Thus the wave equations cannot be applied in our case, including probability interpretation. Though the quarks' behavior in hadrons has a casual nature and the definite metric of the E-space enables to precede straight forward to the probability characteristics.

\section{Free scalar particle}

The model is oriented towards the inside hadronic space, in which a particle cannot be free, so this part is of a methodic character.

Let us introduce the probabilistic space indexed by $\mathbf{E}$-elements and defined by three quantities $(\Omega, \Sigma \mu)$ where $\Omega$ is a multitude of eve, $\Sigma \sigma$ is algebra of $\Omega$ subsets and $\mu$ is a positive measure normalized, and $\mu(\Omega)$ $\leq 1$. If $X_{\mathrm{E}}$ is the real random variable and $\mathrm{X}_{A} \in \Omega$, then the distribution of $\mathrm{X}_{\mathrm{E}}$ is the probabilistic measure on $\Omega \mu=\mathrm{P}\left(\mathrm{X}_{\mathrm{E} 0}<\mathrm{X}_{\mathrm{E}}<\mathrm{X}_{\mathrm{E}}\right)$

Definition 2: The state of the particle is described by the function $\hat{O}\left(x_{E 0}, \mathbf{x}_{E}\right)=1-\mu=\bar{\mu}$, belonging to $\mathbf{E}$ and selected for $\Phi\left(x_{E 0}=\infty, \mathbf{x}_{E}=\infty\right)=0$.

If the functions $\varphi\left(x_{E 0,} \mathbf{x}_{E}\right)=\Phi\left(x_{E}\right)$ describe a scalar particle then its Lagrangian will equal Ò

$$
L=\frac{1}{2}\left(\partial_{\mu} \varphi\right)^{2}+\frac{m^{2}}{2} \varphi^{2},
$$

From which in a usual way we can get a Klein-Gordon-Fock equation in the $\mathbf{E}$ - space

$$
\left(\partial_{0}^{2}+\Delta\right) \varphi\left(x_{E 0,} \mathbf{x}_{E}\right)=m^{2} \varphi\left(x_{E 0}, \mathbf{x}_{E}\right),
$$

Correspondingly to the (3.4)

The obvious function $\varphi\left(x_{E 0}, \mathbf{x}_{E}\right)=\exp \left[-\left(p_{E 0} x_{E 0}+\mathbf{p}_{E} \mathbf{x}_{E}\right)\right]$ may not seem to be the solution of the (4.2), as it will give the conditional expectation value $E\left(\mathbf{x}_{E} \mid x_{E 0}=0\right)=\mathbf{p}_{E}^{-1}$. Under $\mathbf{P}_{\mathrm{E}}=0$ we obtain the nonphysical value $E\left(\mathbf{x}_{E} \mid x_{E 0}=0, \mathbf{v}_{E}=0\right)=\infty$. It is also impossible to use the transition to $K$-representation through a Fourier transformation, as the frequency $k_{E 0}$ and the wave vector $\mathbf{K}_{\mathrm{E}}$ not satisfy the (3.4).

Despite the time coordinate, (4.2) describes the static state. However the infinite velocity in $\mathbf{E}$ - space, makes it possible to transform the equation for the description of dynamic systems. Now represent $\varphi\left(x_{E 0}, \mathbf{x}_{E}\right)$ as the product of two functions $\varphi\left(x_{E 0}, \mathbf{x}_{E}\right)=$ $\varphi_{1}\left({ }_{E 0}\right) \varphi_{\mathrm{rr}}\left({ }_{E}\right)$, each depending on just one variable. Such separation has the following physical meaning. For $\mathbf{v}_{E}=0$ the function $\varphi_{1}\left(x_{E 0}\right)=\varphi\left(x_{E 0}, \mathbf{x}_{E}\right)_{\mathbf{x}_{E}=0}$ will describes the localization phase, and the function $\varphi_{\mathrm{tr}}\left(\mathbf{x}_{E}\right)=\varphi\left(x_{E 0}, \mathbf{x}_{E}\right)_{x_{E 0}=0}$ the translation phase for $\mathbf{v}_{E}=\infty$. These phases cannot exist simultaneously, and supposing the average duration of the localization phase is $\tau_{E}=\left\langle x_{E 0}\right\rangle$, and that of the translation phase is - $\chi_{E}=\left\langle\mathbf{x}_{E}\right\rangle$, and the average phase change occurring with $\tau_{E}$ and $\chi_{E}$, then after every cycle of phase change we get the motion of the particle at the average velocity of $\left\langle\mathbf{v}_{E}\right\rangle=\chi_{E} / \tau_{E}$. Such a separation is due to the infinite velocity.,

Probabilistic approach in compliance with definition 2, consider $\hat{O}\left(x_{E 0}, \mathbf{x}_{\mathrm{E}}\right)=\mathrm{P}\left(\mathrm{X}_{E 0>} \mathbf{x}_{E 0}, \mathbf{X}_{E}>\mathbf{x}_{E}\right)$ being the multidimensional random vector. The random projection of this vector on, for example, axis $x_{\mathrm{E} 0}$ defines the probability of event $P\left(X_{F 0}>x_{F 0}\right)$ and requires the condition $P\left(\boldsymbol{X}_{E}>\mathbf{x}_{\mathrm{E}}\right)=1$ to be commonly met. The latter condition is met for $\mathbf{X}_{\mathrm{E}}=0$ that is $\varphi_{1}\left(x_{E 0}\right)=P\left(X_{E 0}>x_{E 0}\right)$. Accordingly, u, $\varphi_{\mathrm{tr}}\left(\mathbf{x}_{E}\right)=P\left(\mathbf{X}_{E}>\mathbf{x}_{E}\right)$ for $P\left(X_{E 0}>x_{E 0}\right)=1$, i.e. $x_{E 0}=0$. As a result, we arrive at (4.2).

Separating the variables it is necessary to take into account that $\tau_{E}$ and $\leftarrow_{E}$ must be a 4 - vectors: $\tau_{E}^{2}+\chi_{E}^{2}=\Theta^{2}$, and $\Theta^{2}$-E-invariant and $\Theta=c\left(x_{E 0}\right) \tau_{E}=c\left(\mathbf{x}_{E}\right) \chi_{E}$, where

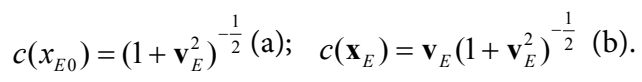

From this it follows that equations for each phase of the i-cycle are to be solutions of (4.1)

$$
\partial_{0}^{2} \varphi_{1}\left(x_{E 0}\right)=k_{E}^{2} \varphi_{1}\left(x_{E 0}\right)(\mathrm{a}) ; \Delta \varphi_{\mathrm{tr}}\left(\mathbf{x}_{E}\right)=\mathbf{k}_{E}^{2} \varphi_{\mathrm{tr}}\left(\mathbf{x}_{E}\right) \quad(\mathrm{b}),
$$

where

$$
k_{E}=m \sqrt{1+\mathbf{v}_{E}^{2}}=E_{E}\left(1+\mathbf{v}_{E}^{2}\right)(\mathrm{a}) ; \quad \mathbf{k}_{A}=\frac{m \sqrt{1+\mathbf{v}_{A}^{2}}}{\mathbf{v}_{E}}(\mathrm{~b}) .
$$

Equation (4.4a) has the following solution $\varphi_{l}\left(x_{E 0}\right)=C_{1} \exp \left(-k_{E} x_{E 0}\right)+C_{2} \exp \left(+k_{E} x_{E 0}\right)$. As attractive potential $\mathrm{V}(r)$ $\geq 0$ equally affects the particle as well as the antiparticle, according to (3.6) $A_{E}=\sqrt{m^{2}-\mathbf{p}_{E}^{2}} \geq 0$ and correspondingly, $K_{\mathrm{E}} \geq 0$. Considering that $x_{E 0} \geq 0$ from Definition 2 it follows that $C_{1}=1, C_{2}=0$, and there remains the decreasing exponent. The probability density $f_{1}\left(x_{E 0}\right)=-d \varphi_{1}\left(x_{E 0}\right) / d x_{E 0}=k_{E} \exp \left(-k_{E} x_{E 0}\right) \quad$ possesses necessary properties: the densities are not negative and the integral of the densities over all values of ${ }_{E 0}$ equals unity. The mathematical expectation of the localization phase duration

$$
\tau_{E}=\frac{1}{k_{E}}=\frac{1}{m \sqrt{1+\mathbf{v}_{E}^{2}}} .
$$

For several cycles, segments $x_{E 0}$ form the simplest stream with no aftereffect. For the free particle in the translation phase the displacement vector of the particle $\mathbf{x}_{E}$ and vector $\mathbf{k}_{A}$ are co-directed and (4.4b) has the following solution

$$
u_{t r}\left(\mathbf{x}_{E}\right)=\widehat{\mathbf{x}}_{E} \exp \left(-\left|\mathbf{k}_{E}\right| x_{E}\right),
$$

Where $x_{E}=\left|\mathbf{x}_{E}\right|$ and $\widehat{\mathbf{x}}_{E}$ is the unit vector. Probability density $f_{t r}\left(\mathbf{x}_{E}\right)=\mathbf{k}_{E} \exp \left(-\left|\mathbf{k}_{E}\right| x_{E}\right)$, i.e. probability density is also positive and the mathematical expectation of the particle displacement in the translation phase is

$$
\chi_{E}=\mathbf{k}_{E}^{-1}=\frac{\mathbf{v}_{E}}{m \sqrt{1+\mathbf{v}_{A}^{2}}} .
$$

As has been assumed, $\tau_{E}$ and $\boldsymbol{\chi}_{E}$ are the components of E-vector and with (2.2)

$$
\tau_{E}^{2}+\chi_{E}^{2}=m^{-2}=\tau_{M l}^{2},
$$

where $\tau_{M l}$ is the average cycle duration in LRF. E-invariant is a value

$$
\tau_{E} p_{E 0}+\chi_{E} \mathbf{p}_{E}=\hbar \text {, }
$$

which equals quantum of action .

On the grounds of (4.10) we consider the cycle duration in LRF $x_{\text {MOl }}$ to be the two-dimensional random vector with random coordinates $x_{E O}$ and $\mathbf{x}_{\mathrm{E}}$, distributed by the exponential law. Then $x_{M O l}$ is also distributed 
by the exponential law $u_{\mathrm{c}}\left(x_{M 0 l}\right)=\exp \left(-m x_{M 0 l}\right)$ with the average value $\left\langle x_{M 0 l}\right\rangle=m^{-1}$. From equality $d \mathbf{x}_{\mathrm{E}}=d \mathbf{x} d \mathbf{x}_{E}=d \mathbf{x}_{M l}$ it follows that $\mathbf{x}_{M l}$ is also distributed by the exponential law $u\left(\mathbf{x}_{M l}\right)=\widehat{\mathbf{x}}_{M l} \exp \left(-\left|\mathrm{k}_{M}\right| x_{M l}\right)$ and $\left\langle\chi_{i l}\right\rangle=\left\langle\chi_{E}\right\rangle$. Using equations (2.3b) and (4.9) we obtain

$$
\chi_{\dot{I} l}\left(\mathbf{v}_{\grave{I}}\right)=\mathbf{v}_{\grave{I}} m^{-1} .
$$

and the average velocity of $\left\langle\mathbf{v}_{M}\right\rangle=\chi_{M l} / \tau_{M l} \leq 1$. The value $\tau_{M l} p_{M 0}-\chi_{M l} \mathbf{p}_{M}=\hbar \sqrt{1-\mathbf{v}_{M}^{2}}$ is also relativistically covariant, and equals to relativistic Lagrangian accurate to a coefficient and changes from $\hbar$ to 0 .

Thus the motion of the particle in E-space is discreet, consists of alternating translation and localization phases and the resultant action for every cycle equals a quantum of motion. The averaged graph of free - particle motion in $\mathbb{E}$ is a random step function with the average step length $\tau_{E}$ and the average step height $\chi_{E}$.

The average duration of free - particle cycle in LRF quantizes time $x_{M 0 l}$ into intervals with the average value $\tau_{M}=m^{-1}$ dependent only on particle mass. And homogeneity is not violated.

\section{Free spinor particle}

Spinor function $\psi\left(x_{E 0} \mathbf{x}_{E}\right)$ also should be a solution to the Dirac equation in $\mathrm{E}$ and describe two phases of motion. To derive the Dirac equation model in $\mathrm{E}$ we need to take into account that $\mathbf{E}=\mathbb{R}_{E}\left(x_{E 0} \geq 0\right) \oplus \mathbb{R}_{E}\left(x_{E 1}, x_{E 2}, x_{E 3}\right)$. The sense of such E-space partition is in the fact that in it the rotation is only possible in $\mathbf{E}=\mathbb{R}_{E}\left(x_{E 0} \geq 0\right) \oplus$ $\mathbb{R}_{E}\left(x_{E 1}, x_{E 2}, x_{E 3}\right)$ and consequently only bispinors have effect. Let us factorize (4.2)

$$
\gamma_{E \mu} \partial_{E \mu} \psi\left(x_{E 0,} \mathbf{x}_{E}\right)=-m \psi\left(x_{E 0,} \mathbf{x}_{E}\right) \text {. }
$$

Matrices $\gamma_{E \mu}$ satisfy the relation $\gamma_{E \mu} \gamma_{E v}+\gamma_{E v} \gamma_{E \mu}=2 g_{E \mu \nu}$, where $g_{E \mu \nu}$ - the Kronecker symbol, and equal

$$
\gamma_{E 0}=\hat{a}=\operatorname{diag}(1,1), \gamma_{E i}=\sigma_{i} .
$$

Function $\psi\left(x_{E 0,}, \mathbf{x}_{E}\right)$ should describe two phases of motion

$$
\psi\left(x_{E 0,} \mathbf{x}_{E}\right)=\psi_{1}\left(x_{E 0}\right) \psi_{\text {tr }}\left(\mathbf{x}_{E}\right) \text {. }
$$

For the localization phase together with (4.5) we obtain

$$
\gamma_{E 0} \partial_{0} \psi_{1}\left(x_{E 0}\right)=-k_{E} \psi_{1}\left(x_{E 0}\right),
$$

where $\psi_{1}\left(x_{E 0}\right)=\beta \exp \left(-k_{E} x_{E 0}\right)$ - bispinor with $k_{E}>0$ and $x_{E 0} \geq 0$.

Equation for the translation phase is

$$
\boldsymbol{\gamma}_{E} \nabla \psi_{\mathrm{tr}}\left(\mathbf{x}_{E}\right)=-\mathbf{k}_{E} \psi_{\mathrm{tr}}\left(\mathbf{x}_{E}\right)
$$

and in compliance with (4.8) the solution is $\psi_{\mathrm{tr}}\left(\mathbf{x}_{E}\right)=\beta \widehat{\mathbf{x}}_{E} \exp \left(-\left|\mathbf{k}_{E}\right| x_{E}\right)$. Then

$$
\boldsymbol{\gamma}_{E} \psi_{\text {tr }}\left(\mathbf{x}_{E}\right)=\frac{\mathbf{k}_{E}}{\left|\mathbf{k}_{E}\right|} \psi_{\text {tr }}\left(\mathbf{x}_{E}\right),
$$

When movement is along axis $x_{3}$

$$
\sigma_{3} \psi_{\text {tr }}\left(x_{E 3}\right)=\frac{k_{E 3}}{\left|k_{E 3}\right|} \psi_{\text {tr }}\left(x_{E 3}\right)
$$

and the space of bispinor $\psi_{\mathrm{tr}}\left(x_{3}\right)$ is a proper space of the diagonal matrix $\sigma_{3}$ with positive and negative helicity and there may be only a discrete transition between these subspaces. The duration of localization phases and the extent of translation phases are defined by formulae (4.7) and (4.11).
All the features of the quantum theory of the scalar particle are valid for spinors as well. But in the latter case we have a new detail of helicity. In $\mathbf{E}$, the helicity of massive fermions is only observed in the translation phase, and it is a "good" quantum number, whereas in $\mathbf{M}$ the helicity of massive fermions with a nonzero mass can't be a quantum number characterizing the particle, since it can be inverted by appropriate Lorentz transformations. Nevertheless, in nature, there exist left and right fermions that are quite different particles and this is seen in $\mathbf{E}$.

\section{Neutral spin or particle in the strong potential}

If the particle is affected by the attractive potential which in the general case equals $\mathrm{V}\left(\mathrm{x}_{0}, \mathbf{x}_{\mathrm{E}}\right)$, then (4.13) will take the form

$$
\gamma_{E \mu} \partial_{E \mu} \psi\left(x_{E 0}, \mathbf{x}_{E}\right)=-\left[m+\mathrm{V}\left(x_{E 0}, \mathbf{x}_{E}\right)\right] \psi\left(x_{E 0}, \mathbf{x}_{E}\right) \partial_{E \mu} \mathrm{V}\left(x_{E 0}, \mathbf{x}_{E}\right) .
$$

If potential $\mathrm{V}\left(\mathbf{x}_{E}\right)$ works then in the localization phase

$$
\gamma_{E 0} \partial_{E 0} \psi_{I V}\left(x_{E 0}\right)=-k_{E}\left(1+\frac{\mathrm{V}\left(\mathbf{x}_{E}\right)}{m}\right) \psi_{I V}\left(x_{E 0}\right) .
$$

The solution to this equation is

$$
\psi_{I V}\left(x_{E 0}\right)=\beta \exp \left(-k_{E}\left(1+\frac{\mathrm{V}\left(\mathbf{x}_{E}\right)}{m}\right) x_{E 0}\right) .
$$

The average duration of the localization phase is

$$
\tau_{\mathrm{V}}=\frac{1}{k_{E}\left(1+\mathrm{V}\left(\mathbf{x}_{E}\right) / m\right)} \text {. }
$$

The equation for the translation form will take the form

$$
\boldsymbol{\gamma}_{E} \nabla \psi_{\mathrm{trV}}\left(\mathbf{x}_{E}\right)=-\mathbf{k}_{E}\left(1+\mathrm{V}\left(\mathbf{x}_{E}\right) / m\right) \psi_{\mathrm{trV}}\left(\mathbf{x}_{E}\right) \nabla \mathrm{V}\left(\mathbf{x}_{E}\right)
$$

and the solution

$$
\psi_{\text {tr }}\left(\mathbf{x}_{E}\right)=\beta \widehat{\mathbf{x}}_{E} \exp \left(-\left(1+\mathrm{V}\left(\mathbf{x}_{E}\right) / m\right)\left|\mathbf{k}_{E}\right| x_{E}\right) .
$$

The average extent of the translation phase is

$$
\chi_{\mathrm{V}}=\frac{1}{\mathbf{k}_{E}(1+\mathrm{V}(\mathbf{x}) / m)} \text {. }
$$
form

With the quantized motion for $\mathrm{V}(r)=c r$ (3.9) takes the following

$$
\mathrm{E}\left(\delta \mathbf{p}_{E q i}\right)=\mathbf{F} \tau_{\mathrm{V} i}(a) ; \quad \mathrm{E}\left(\delta E_{E q i}\right)=-\mathbf{F} \chi_{V_{i}}(b) .
$$

Equation $(4.27 b)$ proves that while the translation phase is on when $x_{\mathrm{E} 0}=$ const, there are instant nonlocal interactions in $\mathbf{E}$. However, when mapped in $\mathbf{M}$ they take place with speed $c$.

\section{Application of the Model of Quantized Motion of Quarcs to Determine Some Properties of Quarks in Protons}

\section{Quantized motion of quarks}

The calculation of the quantized motion of quarks has been done on the basis of the IVC (Figure 1) on the assumption that the quark moves along the axis $z$ which passes though the centre of the proton, parameters of motion $\tau_{\mathrm{v} i}$ and $\chi_{\mathrm{V} z i}$ being of average value. The following data are used in the calculation: root-mean-square radius of the proton $r_{\mathrm{p}}=0.84 \mathrm{fm}$ and $a_{\mathrm{cly}}^{-1}=r_{p}=0.84 \mathrm{fm}$, averaged constituent mass $u$ and $d$ of quarks $0.33 \mathrm{Gev}$. This mass is included into the calculation as Compton wave-length of a quark $\lambda_{q} \approx 0.6 \mathrm{fm}$. The motion of a quark is divided into deceleration and acceleration portions. The 
initial point for the calculation (point A) is chosen at the beginning of the deceleration portion when a quark has passed through the centre of a proton and at point $x_{E}=0, z=\lambda_{q} / 2=0,3 \mathrm{fm}$ the quark localization phase starts. The acceleration portion starts with the translation phase at point $\mathrm{B}$ when $v_{\mathrm{Ez}}=0$ and the end of the translation phase coordinate $x_{E 0}$ has become more than $0.84 \mathrm{fm}$.

The quark deceleration in the second half-period of oscillation starts also with the localization phase at point $\mathrm{C}$ for $v_{\mathrm{Fz}}=\infty$ and $z<0$ and the calculation is done in the way similar to the first half-period. Here the following peculiarity is disclosed: the coordinates of the beginning of the second oscillation $(0.08 \mathrm{fm} 0.32 \mathrm{fm})$ are close to the accepted coordinates of the beginning of the first oscillation $(0.0,0.3 \mathrm{fm})$.

\section{Charge distribution in the proton}

Central-symmetric motion of quarks (Section 3.1) makes it possible to confine to the calculation of the charge distribution for one quark considering that its charge equals the charge of a proton. The calculation is done on the assumption that $\mathrm{V}(r)=c r$ and the charge distribution is defined by the probability of the quark being at a given point of radius $r=|Z|$ and this probability must be determined from the M-space "viewpoint"|

$P\left(r_{i}\right)=\tau_{M I N i} / \sum \tau_{M I N i}$, where $\tau_{M I N i}=\sqrt{\left(\tau_{E V i}\right)^{2}+\chi_{V i}^{2}}$. As the calculations show that the second oscillation practically repeats the first oscillation the parameters of the first oscillation are accepted as the calculation basis. The calculation of the charge density has been done under the condition that the charge is located in the spherical layer with a unit thickness which has radius $r$. After the approximation by the exponential function the equation for the charge density calculation is obtained $\rho_{c}(r)=4.0 \exp (-3.9 r) e / \mathrm{fm}^{3}$ for validity factor $R^{2}=0.85$. The calculated charge distribution along the radius is $j_{c}(r)=4 \pi r^{2} \rho_{c}(r) e / f m$ (Figure 2).

For the comparison the experimental data for the electric formfactor of the proton have been used which are usually described by dipole approximation $G=\left(1+q^{2} / 0.71\right)^{-2}$ [16] for the preset square of 4-momentum $\mathrm{q}^{2}$. This dependence gives the experimental value of charge density $\rho_{e}(r)=3.0 \exp (-4.35 r) e / \mathrm{fm}^{3}$ and that of the distribution of a charge along the radius $j_{e}(r)=4 \pi r^{2} \rho_{e}(r) e / \mathrm{fm}$ (Figure 2).

Graph $j_{c}(r)$ systematically exceeds $j_{e}(r)$. It is connected with the fact that definitional domain $j_{c}(r)$ equals $0<r<0.85 \mathrm{fm}$ and the box under $j_{c}(r)$ equals $\approx 1$. Definitional domain $j_{c}(r)$ equals $0<r<\infty$ and the box

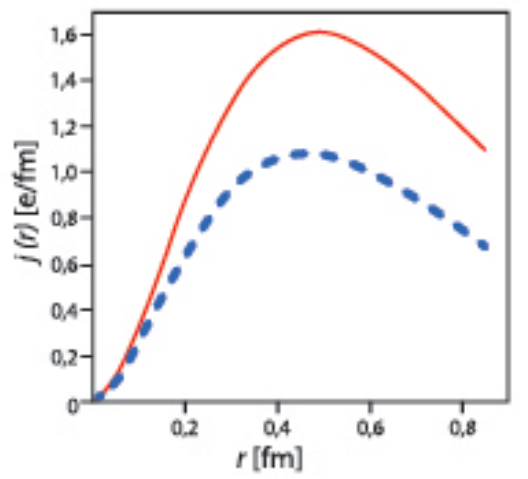

Figure 2: Electric charge distribution in the proton: continuous line is for calculation data, dashlineis for experimental data. under this curve on the section $0<r<0.9 \mathrm{fm}$ equals 0.6.

\section{Conclusion}

It is stated that in the E-space model, Radius-vector rotations group does not mix temporal and spatial coordinates;- kinetic energy diminishes when the speed grows. This determines the existence of constituent and current quarks and describes the dynamic relation of their masses; - to describe quantum movement in the E-space, wave equations cannot be applied. The application of the random function theory has shown that the quarks' movement consists of localization and translation phases;- helicity of massive fermions can be observed only during translation phase and is a "good" quantum number;an infinite velocity and non-local interactions connected with it while mapping in the $\mathbf{M}$-space does not upset the RS-principles: the maximum interaction transmission velocity and the maintenance of causality principle; -the proton charge calculation result plausibly agrees with the experimental data; the four-dimensional values in the E-space are the 4-vector with scalar invariants which have analogies in the $\mathbf{M}$-space;- the E-invariant models have a transparent physical content and are no alternative for the existing QCD methods, but expand their possibilities.

\section{References}

1. Dorokhov A, Tomio L (2000) Pion structure function within the instanton model. Phys Rev.

2. Dorokhov A, Esaibegyan S, Mikhailov S (2000) Eur J Phys C 13: 331

3. Dosch H (1987) condensate and effective linear potential. Phys Lett B 190: 177.

4. Dosch H, Simonov YU (1988) Gluon The area law of the Wilson loop and vacuum field correlators. Phys Lett B 205:339.

5. Bakulev B, Mikhailov S, Stefanis N (2001) Erratum to QCD-based pion distribution amplitudes confronting experimental data. Phys Lett B 508: 279.

6. DElia M, DiGiacomo A, Meggiolaro E (1999) phys Rev D 59.

7. Bakulev A, Mikhailov S (2002) Lattice measurements of nonlocal quark condensates, vacuum correlation length, and pion distribution amplitude in QCD. Phys Rev D 65: 114511.

8. Cornwall J (1982) Dynamical mass generation in continuum quantum chromo dynamics. Phys Rev D 26: 1453.

9. Halzen G, Krein A, Natale A (1993) Relating the QCD Pomeron to an effective gluon mass. Phys Rev D 47: 295

10. Bernard C (1982) Monte Carlo evaluation of the effective gluon mass. Phys Lett B 108: 431.

11. Mandula J, Ogilvie O (1987) The gluon is massive: A lattice calculation of the gluon propagator in the Landau gauge. Phys Lett B 185: 127

12. Djordjevic M, Gyulassy M (2003) Phys Lett B 560.

13. Djordjevic M (2006) Transition radiation in QCD matter. Phy Rev C 73: 044912

14. Kuzmenko D, Simonov YU

15. Alexandrou C, de Forcrand PH, Jahn O (2003) Nucl Phys Proc Suppl 119 667- 669.

16. Belkov A, Kovalenko S (1987) PEPAN 18: 110. 